\title{
Original
}

\section{Prevalencia de anticuerpos contra virus dengue en el cantón de Golfito (2005) y en el Distrito Central de Puntarenas (2005-2006), Costa Rica}

\author{
(Dengue seroprevalence in Golfito and the central district \\ of Puntarenas, Costa Rica 2005-2006)
}

Margarita Lee-Lui, Jonathan Alfaro-Alvarado,Aryam Quesada-Johnson, Lizeth Taylor-Castillo Laya Hun-Opfer

\section{Resumen}

Centro de Investigaciones en EnfermedadesTropicales (CIET). Facultad de Microbiología. Universidad de Costa Rica

Abreviaturas: $\quad A D E$, potenciación dependiente de anticuerpos; CCP, Centro Centroamericano

de

Poblaciones; DEN-2, DEN3, Dengue 3; DEN-4, Dengue 4; Dengue 2; EPI (Expanded Programme on Immunization) DEN-I, Dengue I; Fc, fracción cristalizable;FD,fiebre de dengue; FHD, fiebre hemorrágica por dengue; INEC Instituto Nacional de Estadísticas y Censos; OMS, Organización Mundial de la Salud; SCD, síndrome de choque por dengue.

Correspondencia: Laya Hun. rlhun@cariari.ucr.ac.cr
Justificación y objetivo: La primo infección con el virus dengue varía desde asintomática hasta cuadros muy severos, como el dengue hemorrágico o el síndrome de choque por dengue. El Distrito Primero de Puntarenas ha sido una de las poblaciones más afectadas desde 1993, cuando aparecieron los primeros brotes, hasta hoy, con una disminución de la incidencia en los últimos años. La región Brunca fue la segunda en incidencia en el país en 1998, y si bien la endemicidad se ha mantenido, no existen estudios epidemiológicos sobre la prevalencia de esta enfermedad; los datos que aporta el Ministerio de Salud corresponden a la incidencia de casos clínicos en un período determinado. El objetivo de este estudio fue determinar la seroprevalencia en el Distrito Primero de Puntarenas y en Golfito para conocer la vulnerabilidad de la población de sufrir fiebre hemorrágica por dengue y analizar la situación asociada a los datos epidemiológicos de estas regiones, tales como edad, sexo y ubicación geográfica, ya que una población susceptible es la que presenta una alta seroprevalencia, unida a condiciones de hacinamiento y alta densidad vectorial.

Métodos: El muestreo se realizó con el método EPI descrito por la OMS. Se recolectaron 210 muestras de suero en cada distrito, se desarrolló y estandarizó una prueba de ELISA de captura de antígeno para la detección de anticuerpos tipo IgG contra dengue, usando como referencia el método de reducción de placas de Dulbecco considerado estándar de oro.

Resultados: La seroprevalencia contra dengue fue del 43.8\% (92) y del 90\% (189) en los distritos de Golfito y Central de Puntarenas, respectivamente, sin diferencias significativas respecto al sexo, la edad y la localidad. Se obtuvo un $83 \%$ y un $95 \%$ de correlación en Golfito y Puntarenas, respectivamente, entre los casos sintomáticos y la serología con el método estandarizado. Un $32 \%$ y un $42 \%$ del total de seropositivos en cada localidad afirmaron no haber padecido fiebre por dengue, lo cual refleja, por tratarse de una muestra representativa, que existe al menos un porcentaje similar en el resto de la población con infección asintomática.

Conclusión: La seropositividad de la población contra el virus dengue en el distrito Central de Puntarenas es muy alta, y aunque Golfito ha sido un distrito con reporte de baja incidencia para dengue desde 1998 hasta la fecha, este estudio demuestra que cerca del $50 \%$ de la población presenta anticuerpos contra el virus, lo que significa un alto riesgo de desarrollar dengue hemorrágico en estas localidades en una futura infección por un serotipo diferente. Por lo tanto, es recomendable que las autoridades de salud realicen cambios en el desarrollo de estrategias 
ISSN 0001-6002/2008/50/3/147-152 Acta Médica Costarricense, (C2008 Colegio de Médicos y Cirujanos integrales con la colaboración de la comunidad, el gobierno y las autoridades de Salud, para de mejorar los programas de prevención y control de la enfermedad.

Descriptores: dengue, dengue hemorrágico, seroprevalencia, síndrome de choque por dengue, epidemiología, vector Aedes aegypti

\section{Abstract}

Justification and objectives: Dengue infection could be asymptomatic or it can produce a mild acute febrile illness, dengue fever, or a severe illness, such as hemorrhagic dengue or shock syndrome (DHF/SSD). Illness severity depends upon various risk factors like viral characteristics, infestation of the vector as well as host factors; presence of more than 1 dengue virus serotype and secondary infections. Since 1993 when the first cases of dengue were reported in Costa Rica Puntarenas's First District population has been 1 of the most affected areas, although there has been a decline in the incidence over the last years. The Brunca Region was the second with high incidence of dengue in 1998 and since then cases have been reported permanently. The national incidence is based on the clinical cases weekly report by the Ministry of Health, but there are no seroepidemyological studies to determine the seroprevalence of the disease. Determining IgG antibodies prevalence against dengue at the First Puntarenas's District and at Golfito district will allow us to better understand the incidence and calculate the percentage of the population at risk of developing DHF/SSD associated with the epidemiological data.

Methods: For the seroprevalence study we collected 210 sera samples from each region and used an IgG capture ELISA developed and standardized in our laboratory using as reference the Dulbecco Plaque Method. Samples were obtained utilizing the EPI method (Expanded Program on Immunization) described by the World health Organization (WHO).

Results: The study showed 43.8\% (92) and 90\% (189) prevalence in the districts of Golfito and Puntarenas respectively, no significant differences in gender, age and geographical location were found. We determined an $83 \%$ in Golfito and a 95\% in Puntarenas correlation between serology and symptomatic cases and $42 \%$ from total seropositive people in each region denied having clinical symptoms. Since this is a representative sample study we can affirm that a similar percentage of asymptomatic cases occur in the general population.

Conclusions: Seropositivity against dengue virus in the First Puntarenas's District population is very high $(90 \%)$ and although the Golfito District has been reported with a low prevalence since 1998 , this study shows that near $50 \%$ of the population is seropositive with the consequent risk for DHF/SSD. It is very important to continue this type of seroepidemiological studies in other regions of the country to verify the real threat of this disease in order to take the necessary preventive measures.

Key words: dengue fever, dengue hemorrhagic fever, seroprevalence, dengue shock syndrome, epidemiology, vector Aedes aegypti

Recibido: 27 de setiembre de 2007
Aceptado: 8 de abril de 2008
El dengue es una enfermedad causada por el virus dengue transmitido por el vector Aedes aegypti en zonas urbanas y por Aedes albopictus en áreas peridomiciliares y silvestres. ${ }^{1}$ Existen cuatro serotipos de dengue: DEN-1, DEN-2, DEN-3 y DEN-4, diferenciables entre sí por las técnicas de reducción de placas de Dulbecco y por el método de reacción en cadena de la polimerasa (PCR). ${ }^{2}$ Se han descrito variantes genotípicas en cada uno de estos serotipos, las cuales están asociadas a zonas geográficas diferentes (topotipos) que presentan mayor o menor virulencia. ${ }^{3} \mathrm{El}$ genotipo Americano (AM) del DEN-2 fue el virus endémico en el hemisferio occidental y el Pacífico del Sur por más de 5 décadas. Sin embargo, no hay evidencia de casos de fiebre hemorrágica por dengue (FHD)/síndrome de choque por dengue (SCD) causados por este genotipo, aun cuando ocurra en infección secundaria. ${ }^{4}$ El genotipo de Sureste de Asia (SEA) fue inicialmente detectado en 1981 en Cuba, ${ }^{3}$ durante una epidemia con miles de casos de FHD reportados. ${ }^{5-7}$ 
Luego se demuestra este mismo genotipo en las epidemias de FHD en México, Perú y Texas, en 2005, ${ }^{5}$ desplazando al genotipo AM. Esto permite entender cómo las cepas virulentas se diseminan y desplazan a las menos virulentas, ${ }^{8}$ lo cual podría explicar el aumento en los casos de dengue hemorrágico en los países endémicos y por ende en Costa Rica.

En el país se erradica el vector en 1960 y desde 1971 se han dado reinfestaciones en Puntarenas, Liberia y Limón. (Dr. Mario Vargas V., Departamento de Parasitología, Facultad de Microbiología, comunicación personal). Sin embargo, el reporte oficial de la reintroducción del vector al territorio se establece hasta 1992..$^{9}$ Datos de la OPS reportan circulación de virus DEN-1 en Cuba, Puerto Rico y Venezuela desde 1977, y se disemina posteriormente a México, América Central y el Norte de Suramérica. ${ }^{5}$ En 1985 el Centro para el Control de Enfermedades de Estados Unidos (CDC) reporta la introducción de Ae. albopictus al hemisferio, ${ }^{5}$ lo cual podría desempeñar un papel muy importante en la epidemiología de dengue, al mantener el ciclo del virus en la naturaleza. Los primeros casos de dengue (DEN-1) se reportaron en Costa Rica en octubre de 1993, en las regiones Pacífico Central y Chorotega, ${ }^{10} \mathrm{y}$ se ha convertido en un problema importante de salud pública, ya que según datos del Ministerio de Salud, el 95\% de la población costarricense está en riesgo de contraer la infección. ${ }^{11-13}$

Desde el punto de vista epidemiológico, es preciso destacar que una población susceptible a FHD/SCD es aquella que presenta alta seroprevalencia y que se encuentra en zonas con alta densidad vectorial, en hacinamiento y con aumento demográfico. ${ }^{5,14,15}$ Desde 1993 se han presentado casos en el país, siendo 2005 el año con el mayor número de casos $(37,798)$; el único serotipo circulante hasta 1997 fue el DEN-1. Durante la epidemia de 1997 circuló principalmente el DEN-3 y en el periodo comprendido entre 2000 y 2001 se identificó el DEN-2. En 2003 y 2004 solo se reportó el serotipo DEN-1 y desde 2005 a la fecha se registran concomitantemente los serotipos 1 y 2. En 1995 se reportó el primer caso de FHD y hasta la semana 48 de 2007 se habían registrado más de quinientos casos. ${ }^{13}$

En el Distrito Primero de Puntarenas se reportaron 5986 casos de dengue entre 1993 y 2006, siendo siempre la zona más afectada de la región Pacífico Central (Ministerio de Salud, sede Puntarenas). Por otra parte, esta es la segunda región con mayor número de casos de FDH (44.4\%) después de la Chorotega (50\%), de los cuales un $57,6 \%$ pertenece al sexo masculino y el grupo de edad más afectado es el de 10 a 39 años (72\%), durante 2006. En 1998 la región Brunca fue la de mayor incidencia de dengue en el país, y de los 852 casos reportados, 826 pertenecen al cantón de Golfito ${ }^{16}$ y representaron el 33\% de los casos en el nivel nacional. En 1999 el número de casos reportados en Golfito se redujo a 77 (1.3\% del total de casos del país); en 2000 se presentaron 142 casos (2.8\% del total). En 2001-2004 la tasa de incidencia en esta localidad se mantiene y a partir de 2005 nuevamente aumenta. ${ }^{13}$
Conocer la prevalencia de anticuerpos IgG contra dengue en Distrito central de Puntarenas y en Golfito (Figura 1), en asociación con los datos epidemiológicos en esta localidad permitirá conocer y evaluar el comportamiento de la prevalencia de la infección por dengue en esas regiones, así como determinar el porcentaje de la población con mayor riesgo de desarrollar dengue hemorrágico.

\section{Materiales y métodos}

Para la selección de la muestra se utilizó el método EPI (Expanded Programme on Immunization) ${ }^{17}$ descrito por la Organización Mundial de la Salud (OMS), el cual estima los valores del estudio con \pm 10 puntos porcentuales con respecto a la proporción real, con un $95 \%$ de confianza y una seroprevalencia esperada del 50\%. El Instituto Nacional de Estadísticas y Censos (INEC) proporcionó el número total de segmentos censales de los distritos de Puntarenas y Golfito, su ubicación específica y la cantidad de hogares y personas en cada uno de ellos. Se realizó un muestreo de 30 conglomerados en dos pasos: selección de cada uno por medio de un muestreo proporcional al tamaño, con la colaboración y recomendación del Centro Centroamericano de Poblaciones (CCP), y selección de la casa de arranque por muestreo simple al azar. Por cada conglomerado se seleccionan siete casas a partir de la vivienda ubicada más al noroeste y se cuenta la cantidad de casas en sentido de las manecillas del reloj para un tamaño total de 210 muestras de suero representativas de cada una de las regiones. Para la selección de la persona a la cual se le tomó la muestra, se recurrió a una tabla de números aleatorios. ${ }^{17}$

El estudio fue aprobado por el Comité Ético Científico de la Vicerrectoría de Investigación de la Universidad de

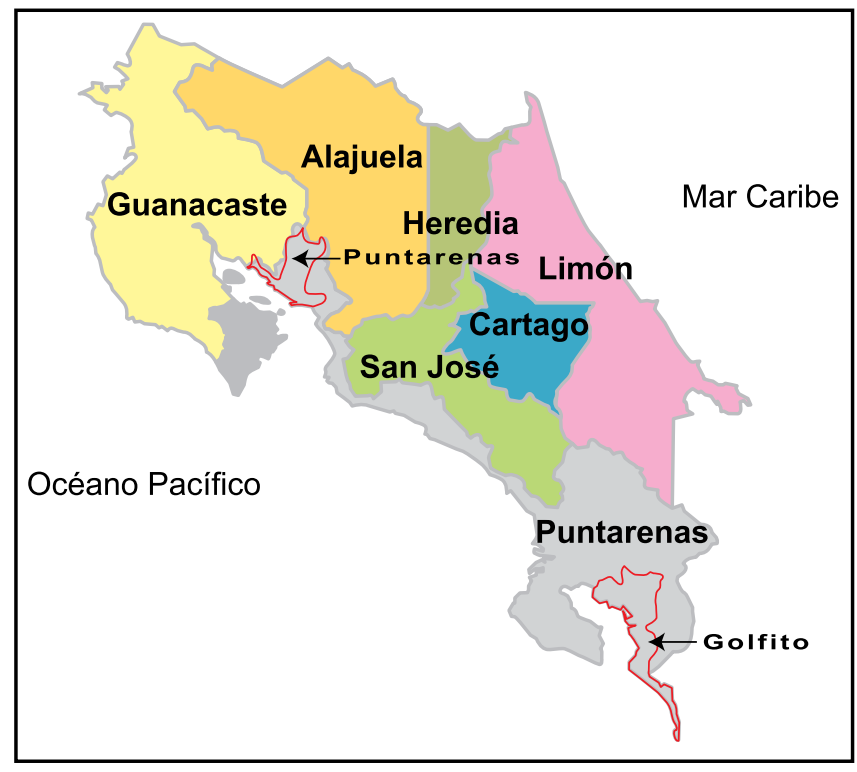

Figura 1. Localización de los distritos Primero de Puntarenas y Golfito, en Costa Rica 
Costa Rica. Cada persona firmó un consentimiento informado y se le realizó una entrevista que incluyó: edad, sexo, años de vivir en el lugar, dónde y cuándo ha viajado fuera del distrito, y si por algún medio se ha dado cuenta de haber padecido dengue en algún momento de su vida (reporte del INCIENSA o por caso clínico, según la Unidad de Salud consultada).

\section{Toma y procesamiento de las muestras de sangre}

Se tomaron $10 \mathrm{ml}$ de sangre por venopunción periférica, se transportó en frío y se obtuvo el suero, el cual se conservó a $-70^{\circ} \mathrm{C}$ hasta su procesamiento.

ELISA: Se diseñó y estandarizó un ensayo inmunoenzimático (ELISA) de captura de antígeno para la detección de anticuerpos tipo IgG contra dengue en la Sección de Virología, utilizando como parámetro de referencia la técnica de placas de Dulbecco, considerada el estándar de oro $^{18}$, donde se alcazó un $100 \%$ de correlación. Se analizaron diferentes diluentes, concentración de muestras, concentraciones de anticuerpos y conjugados, soluciones bloqueadoras y condiciones de incubación, hasta conseguir las características óptimas. ${ }^{19}$ Brevemente, $100 \mu \mathrm{l}$ de la muestra de suero de cada paciente diluida 1:40 se incuba una hora a $37^{\circ} \mathrm{C}$ en placas recubiertas con lisados de los cuatro virus de dengue capturados con anticuerpos de líquido ascítico hiperinmune (donado por el Instituto de Medicina Tropical Pedro Kouri de Cuba, IPK). Después de 3 lavados, se agregan $100 \mu \mathrm{l}$ de conjugado anti-IgG peroxidasa hecho en cabra (Sigma) diluido 1:30000 y se incuba de la misma manera. Se lava nuevamente y se agrega el sustrato líquido TMB (Sigma). Después de 20 minutos se detiene la reacción con $\mathrm{H}_{2} \mathrm{SO}_{4} 1 \mathrm{M}$ y se lee la absorbancia a $450 \mathrm{~nm}$. Se utilizaron como controles positivos y negativos 2 muestras antes caracterizadas en el INCIENSA.

Análisis estadísticos: Se determinó el porcentaje de seroprevalencia y se realizaron pruebas de chi-cuadrado con

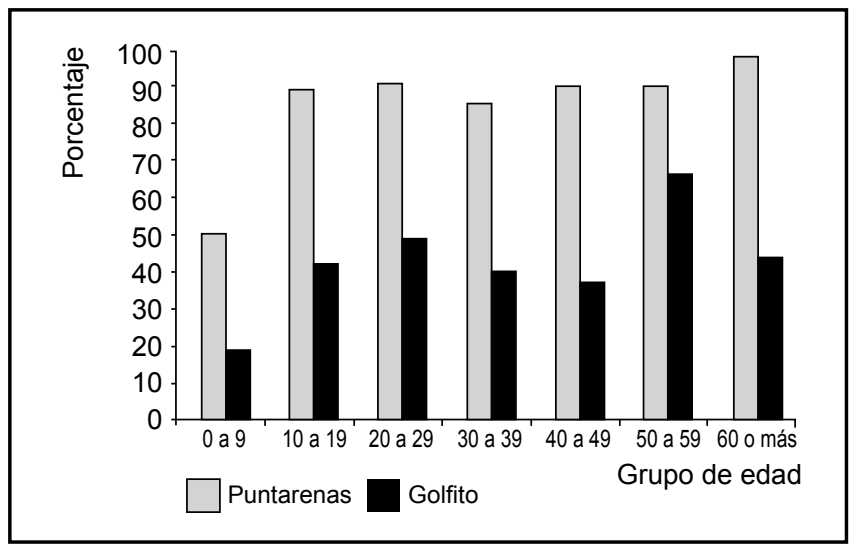

Figura 2. Seroprevalencia de dengue, según grupo etario en los distritos Primero de Puntarenas y Golfito un $95 \%$ de intervalos de confianza para comparar las variables cualitativas con respecto al sexo, edad y distribución por regiones.

\section{Resultados}

La prevalencia de anticuerpos contra los virus de dengue fue de un $43.8 \%$ (92) y de un 90\% (189) en muestras del distrito de Golfito y del Distrito Primero de Puntarenas respectivamente. La distribución en grupos etarios fue muy semejante desde los 10 hasta los 59 años (no se determina diferencia significativa, $p>0.05$ con un $95 \%$ de intervalos de confianza), pero fue menor en los niños de 0 a 9 años, y mayor en Golfito para el grupo de 50 a 59 años (figura 2).

El $55.7 \%$ (117) y el $59.5 \%$ (125) de las personas analizadas pertenecían al sexo femenino en ambas localidades, con un $52 \%$ y un $60 \%$ de positivas por anticuerpos contra el virus, respectivamente, sin embargo, no hubo diferencias significativas con respecto al grupo de varones ( $p>0.05$ con $95 \%$ de intervalos de confianza). De igual forma, no se encontró diferencia con la distribución por localidad en cada distrito con el método de $x^{2}$.

La asociación entre los antecedentes clínicos de dengue y los resultados del ELISA fue de un $83.3 \%$ y un $95 \%$ para Golfito y Puntarenas, respectivamente, siendo mayor el número de positivos sin síntomas en el distrito de Puntarenas (figura 3). Los casos asintomáticos fueron identificados en un $32 \%$ y un $42 \%$, lo cual, aunado a los que reportan sintomatología asociada sin asistencia a un Centro de Salud, arroja un total del $38 \%$ al $68 \%$ de infecciones que no son registradas.

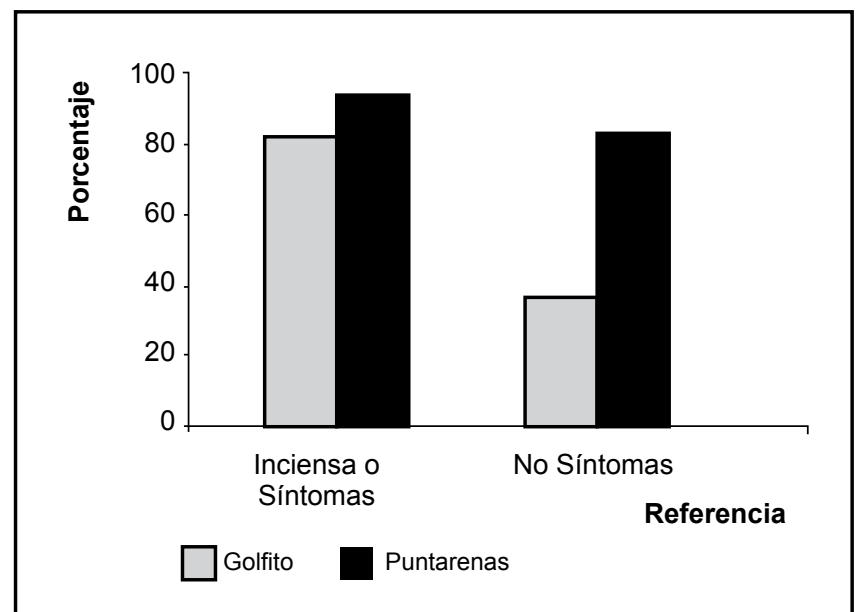

Figura 3. Distribución del total de positivos obtenidos de la prueba de ELISA, según referencias obtenidas de los encuestados de haber o no padecido de dengue. 


\section{Discusión}

La situación en Costa Rica es preocupante, las regiones de mayor riesgo (Huetar Atlántica, Chorotega y Pacífico Central) hacen evidente su vulnerabilidad por el incremento de casos por FD en 2007. Son 15 años de lucha contra el dengue con la particularidad de que la cantidad de casos por FHD desde 1993 hasta principios de 2007, había sido baja en comparación con otros países (40 casos en total). Pero, solo en 2007 se presentó un total de 311 casos de FHD (Ministerio de Salud, 2007). Una posible razón de la baja prevalencia en años anteriores es el hecho de que los serotipos cocirculantes en algunas regiones del país son DENV-1 y DENV-2, según reportes de INCIENSA, y que las infecciones secundarias ocurran lo suficientemente cercanas a las infecciones primarias, lo que disminuye la probabilidad del desarrollo de FHD. Por otra parte, se debe considerar la nueva población en las zonas de riesgo, el alto número de casos asintomáticos (figura 3 ) y la posibilidad de que los genotipos de los serotipos circulantes sean de baja virulencia.

En el estudio la disminución del número de casos por FD en el Distrito Primero de Puntarenas y en Golfito, en relación con años anteriores, podría explicarse con la alta seroprevalencia de la población contra DENV, lo que indica que las infecciones son constantes, pero asintomáticas. Por lo tanto, la disminución del número de casos reportados no se debe solamente a las campañas o programas de prevención realizados, sino también a la inmunidad de la población. A pesar de que la transmisión del virus es controlada en los periodos de las epidemias, se podría producir una falsa seguridad al bajar la incidencia de dengue clásico, pero no así del riesgo a desarrollar $\mathrm{FHD} / \mathrm{SCD}$. Esta población que desconoce su estatus inmune representa una porción importante de la comunidad que posee dicho factor de riesgo, en especial en Golfito, donde no se ha reportado cocirculación de virus. Según el Dr. Pedro Kouri (comunicación personal), se recomienda multiplicar por 5 ó 10 el número de casos reportados por FD para obtener un aproximado del total de la población seropositiva. Si este es el caso, en Puntarenas por ejemplo, se sobrepasa el total de la población del distrito (9559 habitantes) al multiplicar los 5.986 casos reportados por 5; esto es debido al desplazamiento continuo de las personas de una zona a otra, alterándose la distribución de la población seropositiva. Por otra parte, en enero de 2008 se reportó el ingreso de dengue 3 por la frontera sur del país ${ }^{20} y$ este nuevo serotipo aumenta el riesgo de una epidemia por FHD, considerando que el desarrollo de FHD/SCD, depende también de otros factores como la virulencia de las cepas circulantes, la condición inmunológica de la población, condiciones individuales, ambientales, y otros factores epidemiológicos, como grado de infestación del vector. ${ }^{5,715}$

Seroprevalencias de otros países como Cuba, México, Ecuador y Nicaragua, son también altas, por ejemplo, cerca del $50 \%$ de la población cubana era seropositiva contra el
DEN-1 para la epidemia de 1977-1978 y contra el DEN-2 en 1981, y dado que se controló al Aedes aegypti, el ciclo de transmisión se detuvo ${ }^{21}$. En 2006, Álvarez determina que en La Habana un $60 \%$ de la población era seropositiva por el DEN-1, y solo un $15 \%$ por el DEN-2. ${ }^{22}$ En Jaltipán, México, se obtuvo un 79,6\% de seropositividad ${ }^{23}$ y en Carbo, Ecuador, se encontró una baja incidencia de casos por FD, pero una alta seropositividad $(58,6 \%)$ destacándose como una zona de alto riesgo por $\mathrm{FHD}^{24}$. En Nicaragua, se estudió una población escolar y se obtuvo más del $90 \%$ de seropositividad en niños mayores de 7 años. ${ }^{25}$ Lo anterior deja claro que la población aquí estudiada no es la única con alta seroprevalencia en comparación con otras poblaciones endémicas. Más bien, se confirma el resultado de zona endémica alcanzado, ya que el Distrito Primero de Puntarenas y el de Golfito, cumplen con muchos de los factores de riesgo ya mencionados.

La distribución de la seroprevalencia según el sexo y la edad no fueron significativamente diferente, lo que puede explicarse por la circulación del mismo serotipo de dengue durante un periodo prolongado, así como por diferentes condiciones de prevención y protección individuales en cada distrito, y es consistente con otros estudios en $\mathrm{Cuba}^{1,21} \mathrm{y}$ en México. ${ }^{23}$ Es importante mencionar que la presencia de casos en el grupo etario de 0 a 9 años es muy preocupante, debido a que la población infantil es más susceptible a presentar complicaciones por aumento en la permeabilidad vascular en infecciones secundarias.

Las diferencias encontradas entre los resultados de las muestras y los reportes de las entrevistas podrían deberse a que en INCIENSA y en nuestro laboratorio se utilizaron diferentes métodos de ELISA. Cabe mencionar además que es posible obtener resultados falsos positivos por serología, debido a reacciones cruzadas con otros flavivirus, como el virus del Oeste del Nilo. Sin embargo, en Costa Rica no existen estudios que documenten la presencia de este agente.

Por la alta prevalencia encontrada es recomendable que las autoridades de Salud, la comunidad tomen las medidas necesarias para el controlar el vector, cumpliendo con los cuatro principios básicos establecidos por la OMS y la OPS: voluntad política de los gobiernos, coordinación intersectorial, participación activa de la comunidad y fortalecimiento de las leyes sanitarias nacionales. Aunque esto resulte todo un reto, trabajar en equipo es posible. Cuba implementó sistemas de diagnóstico oportuno según las manifestaciones clínicas del paciente, sobre todo dolor epigástrico y vómito, para darle mayor atención y así evitar su evolución a un FHD e impedir que el ciclo de transmisión del virus continúe. ${ }^{2}$ La determinación de albúmina en pacientes graves por FD también puede ayudar en el pronóstico de FHD y así asistir con rápidez al paciente con reposición de fluidos y electrolitos. ${ }^{26} \mathrm{El}$ estudio a través de proyectos de investigación que permitan determinar cuáles serotipos circulan en el vector, cuál es la densidad del vector, junto con el mejoramiento de la organización integral de la comunidad, del gobierno y de los Centros de Salud, por 
ejemplo, con un aumento del número de cuadrillas en cada comunidad, promovería el desarrollo de un programa preventivo eficaz. ${ }^{27}$ Un aporte de mucho valor sería que la Universidad de Costa Rica se integrara de manera más activa a las campañas de prevención, ayudando a determinar la seroprevalencia en otras poblaciones de alto riesgo; de esta manera los programas de prevención serían mejor dirigidos a regiones que necesitan mayor atención. En un futuro se realizarán estudios en los cuales se pretende capturar las hembras del mosquito vector del dengue, con el fin de determinar el índice de infestación y verificar los serotipos y genotipos de virus de dengue que circulan en el país.

El conocimiento adecuado de la enfermedad y el desarrollo de medidas preventivas en la comunidad no bastan; el éxito de las estrategias dependen de un cambio de conciencia junto con la participación activa de la sociedad, de los Centros de Salud y del gobierno.

Fuente de apoyo. Vicerrectoría de Investigación, Universidad de Costa Rica. Proyectos de Investigación: 803-A5-326, 803-A6-314.

\section{Referencias}

1. Gubler DJ. Dengue and Dengue Hemorrhagic Fever. Clin Microbiol 1998; 11:480-496.

2. Lanciotti R, Calisher C, Gubler D, Chang G, Vorndam V. Rapid detection and typing of dengue viruses from clinical samples by using Reverse Transcriptase Polymerase Chain Reaction. J. Clin Microbiol 1992; 545-554.

3. Rico-Hesse R, Harrisosn LM, Salas RA, Tovar D, Nisalak A, Ramos C. et al. Origins of dengue type 2 associated with increased pathogenicity in the Americas. Virology 1997; 230:244-251.

4. Clyde K, Kyle JL, Harris E. Recent Advances in deciphering viral and host determinants of dengue virus replication and pathogenesis. J Virol 2006; 80:11418-11431.

5. Guzman MG, Kouri GH. Dengue and dengue hemorrhagic fever in the Americas: lessons and challenges. J Clin Virol 2003; 27:1-13.

6. Kochel TJ, Watts DM, Gozalo AS, Ewing DF, Porter KR, Russell KL.Cross-serotype neutralization of dengue virus in Aotus nancymae monkeys. J Infect Dis 2005; 15: 1000-1004.

7. Carrington C, Foster J, Pybus O, Bennet S, Holmes E. Invasion and maintenance of dengue virus type 2 and type 4 in the Americas. J. Virol 2005; 79:14680-14687.

8. Anderson J, Rico- Hesse R. Aedes aegypti vectorial capacity is determined by the infecting genotype of dengue virus. Am J Trop Med Hyg 2006; 75:886-892.

9. Ministerio de Salud, Caja Costarricense de Seguro Social y Organización Panamericana de la Salud Informe Final "Estrategia Nacional de Gestión Integrada de Prevención y Control del Dengue en Costa Rica" periodo 28 de noviembre al 1 de diciembre de 2004.

10. World Health Organization. Outbreak of classic dengue, Costa Rica. Wkly Epidemiol Rec. 1994; 69:85-86.Ministerio de Salud Pública de Costa Rica. Comisión Técnica Interinstitucional. Normas técnicas para el control del dengue y dengue hemorrágico. Costa Rica 2000.
11. Ministerio de Salud de Costa Rica. Análisis Sectorial de Salud: Componente Demográfico epidemiológico. 2006.

12. Iturrino-Monge R, Avila-Agüero ML, Avila-Agüero CR, Moya-Moya $\mathrm{T}$, Cañas-Coto A, Camacho-Badilla K, Zambrano-Mora B. Seroprevalence of dengue virus antibodies in asymptomatic Costa Rican children, 2002-2003: a pilot study. Rev Panam Salud Pública. 2006; 20:39-43.

13. Ministerio de Salud de Costa Rica. Boletines estadísticos de enfermedades de declaración obligatoria. 1999-2007.

14. Kliks S, Nisalak A, Brandt W, Wahl L, Burke D. Antibody -Dependent enhancement of dengue virus growth in human monocites as a risk factor for dengue hemorrhagic fever. Am J Trop Med Hyg 1989; 440:444-451.

15. Halstead SB. Observations related to pathogenesis of dengue hemorrhagic fever. VI. J Biol Med 1970; 42:350-362.

16. Sáenz E, Maranda L,Piza J, González L, Achoy R. Evaluación del sistema de vigilancia epidemiológico del dengue utilizando como indicador la aplicación de la definición de caso sospechoso, Costa Rica 1998. Rev Costarric Cienc Méd 2001; 22:117.129.

17. Hoshaw S. Description and comparison of the methods of cluster sampling and lot quality assurance sampling to assess immunization coverage. Department of vaccine and biological, World Health Organization, Geneva. 2001.

18. Rusell PK, Nisalak A, Sukhavachana P, Vivona S. A plate reducction test for dengue virus neutralizing antibodies. J Immunol 1967; 99: 285-290.

19. Lee M. Estudio seroepidemiológico de dengue en el distrito de Golfito, Costa Rica, en el año 2005. Trabajo de Graduación para optar por el grado de licenciatura en Microbiología y Química Clínica. Junio. 2006.

20. Ávalos A. Salud detecta nuevo tipo de dengue en la frontera sur. La Nación; 25 de enero 2008.

21. Kourí G, Guzmán MG, Valdés L, Carbonel I, del Rosario D, Vázquez S, Laferté J, Delgado J, Cabrera MV. Reemergence of dengue in Cuba: a 1997 epidemic in Santiago de Cuba. Emerg Infect Dis 1998; 4:89-92.

22. Álvarez M, Rodríguez-Roche R, Bernardo L, Vázquez S, Morier L, González D, Castro O, Kouri G, Halstead SB, Guzmán MG. Dengue hemorrhagic fever caused by sequential dengue 1-3 virus infection over a long time interval: Havana Epidemic, 2001-2002. Am J Trop Med Hyg 2006; 75:1113-7.

23. Navarrete J, Acevedo J, Huerta E, Torres J, Gavaldón, D. Prevalencia de anticuerpos contra dengue y leptospira en la población de Jaltipan, Veracruz. Salud Publica Mex 2006; 48:220-228.

24. Cotto R, Avala A, Mosquera C, Russell K, Watt D. Seroprevalencia de dengue en la ciudad de Guayaquil y Durán, Ecuador, Abril 2000. Médicos-Ecuador 2000-2004 en línea: www.medicosecuador.com

25. Balsameda A, et al. High seroprevalence of antibodies against dengue virus in a prospective study of schoolchildren in Managua, Nicaragua. Trop Med Int Health 2006; 2: 935-942.

26. Clyde K, Kyle J, Harris E. Minireview. Recente Advances in Deciphering Viral and Host Determinants of Dengue Virus Replication and Pathogenesis. J Virol 2006: 11418-11431.

27. Kouri G. El Dengue, un problema creciente de salud en las Américas. Rev Panam Salud Pública 2006; 19:143-145. 